OPEN ACCESS

Edited by:

Binod Kumar,

Loyola University Chicago,

United States

Reviewed by:

Rupkatha Mukhopadhyay, Johns Hopkins Medicine,

United States

Raphael Gaudin,

UMR9004 Institut de Recherche en

Infectiologie de Montpellier

(IRIM), France

${ }^{*}$ Correspondence:

Leticia A. M. Carneiro

leticiac@micro.ufri.br

Specialty section:

This article was submitted to Virus and Host,

a section of the journal

Frontiers in Cellular

and Infection Microbiology

Received: 15 February 2021

Accepted: 15 April 2021

Published: 28 April 2021

Citation:

Prestes EB, Bruno JCP, Travassos LH and Carneiro LAM (2021) The Unfolded Protein Response and Autophagy on the Crossroads of

Coronaviruses Infections.

Front. Cell. Infect. Microbiol. 11:668034.

doi: 10.3389/fcimb.2021.668034

\section{The Unfolded Protein Response and Autophagy on the Crossroads of Coronaviruses Infections}

\author{
Elisa B. Prestes ${ }^{1}$, Julia C. P. Bruno ${ }^{2}$, Leonardo H. Travassos $^{3}$ and Leticia A. M. Carneiro ${ }^{2 *}$ \\ 1 Institut Necker Enfants Malades, Université Paris Descartes, Paris, France, ${ }^{2}$ Laboratório de Inflamação e Imunidade, \\ Instituto de Microbiologia Paulo de Goes, Universidade Federal do Rio de Janeiro, Rio de Janeiro, Brazil, ${ }^{3}$ Laboratório de \\ Imunoreceptores e Sinalização Celular, Instituto de Biofísica Carlos Chagas Filho, Universidade Federal do Rio de Janeiro, \\ Rio de Janeiro, Brazil
}

The ability to sense and adequately respond to variable environmental conditions is central for cellular and organismal homeostasis. Eukaryotic cells are equipped with highly conserved stress-response mechanisms that support cellular function when homeostasis is compromised, promoting survival. Two such mechanisms - the unfolded protein response (UPR) and autophagy - are involved in the cellular response to perturbations in the endoplasmic reticulum, in calcium homeostasis, in cellular energy or redox status. Each of them operates through conserved signaling pathways to promote cellular adaptations that include re-programming transcription of genes and translation of new proteins and degradation of cellular components. In addition to their specific functions, it is becoming increasingly clear that these pathways intersect in many ways in different contexts of cellular stress. Viral infections are a major cause of cellular stress as many cellular functions are coopted to support viral replication. Both UPR and autophagy are induced upon infection with many different viruses with varying outcomes - in some instances controlling infection while in others supporting viral replication and infection. The role of UPR and autophagy in response to coronavirus infection has been a matter of debate in the last decade. It has been suggested that CoV exploit components of autophagy machinery and UPR to generate double-membrane vesicles where it establishes its replicative niche and to control the balance between cell death and survival during infection. Even though the molecular mechanisms are not fully elucidated, it is clear that UPR and autophagy are intimately associated during CoV infections. The current SARS-CoV-2 pandemic has brought renewed interest to this topic as several drugs known to modulate autophagy - including chloroquine, niclosamide, valinomycin, and spermine - were proposed as therapeutic options. Their efficacy is still debatable, highlighting the need to better understand the molecular interactions between CoV, UPR and autophagy.

Keywords: coronavirus, autophagy, unfolded protein response, integrated stress response, hostpathogen interaction 


\section{INTRODUCTION}

From single to multicellular, every living organism is frequently exposed to variable environmental conditions - including extremes of temperature, nutrient deprivation, irradiation, hypoxia, infections, and others - that can result in cell damage and/or dysfunction (Galluzzi et al., 2018). Eukaryotic cells have evolved mechanisms to cope with the stress generated by these conditions and support cellular functions, thereby maintaining microenvironmental and organismal homeostasis (Galluzzi et al., 2018). Such mechanisms include the DNA damage response (Jackson and Bartek, 2009), mitochondrial stress signaling (Chang et al., 2017), autophagy (Galluzzi et al., 2017) and the unfolded protein response (UPR), which is a component of the integrated stress response (Galluzzi et al., 2018) (ISR). The ISR is an evolutionarily conserved intracellular signaling network that can be initiated by several types of stress and converges to a common signaling hub - the phosphorylation of the $\alpha$-subunit of the eukaryotic translation initiation factor 2, eIF2 $\alpha$ (CostaMattioli and Walter, 2020). A family of four eIF2 $\alpha$-kinases is capable of sensing alterations in cellular homeostasis and respond by phosphorylating eIF2 $\alpha$ : (i) double-stranded RNA (dsRNA)-dependent protein kinase (PKR) that is activated mainly by dsRNA during viral infection but also by oxidative and endoplasmic reticulum (ER) stress, growth factor deprivation, cytokines, bacterial infections, and ribotoxic stress (García et al., 2006); (ii) PKR-like ER protein kinase (PERK), which senses ER stress and also perturbations in calcium homeostasis, cellular energy or redox status (McQuiston and Diehl, 2017); (iii) heme-regulated eIF2 $\alpha$ kinase (HRI), a sensor for low levels of intracellular heme as well as the formation of cytosolic protein aggregates, arsenite-induced oxidative stress, heat shock, nitric oxide, $26 \mathrm{~S}$ proteasome inhibition, and osmotic stress (Girardin et al., 2020); and (iv) general control nonderepressible 2 (GCN2) that is activated in response to amino acid deprivation when it binds to deacylated transfer RNAs (tRNAs) via histidyl-tRNA synthetase-related domain (Battu et al., 2017). Not only do each of these pathways initiate crucial re-programming of the cell by modulating transcription of key genes and translation of new proteins, they also intersect with other stress-response pathways to restore homeostasis. Although ISR is primarily a homeostatic-preserving program by which cells adapt to survive, severe or long-lasting stress can induce cell death signaling by regulating autophagy or apoptosis.

The UPR is the cellular response to disturbances in the ER that ensues when its folding capacity is exceeded and unfolded proteins accumulate (Hetz, 2012). The ER is the primary site for the synthesis and folding of secreted and transmembrane proteins in eukaryotic cells. A myriad of environmental conditions (including nutrient deprivation, hypoxia, and loss of calcium homeostasis) can significantly alter the amount of proteins entering the ER, leading to UPR activation by one or more of three conserved ER-stress sensors: PERK, X-boxbinding protein 1 (XBP-1) and activating transcriptional factor-6 (ATF-6) (Siqueira et al., 2018). These sensors display ER luminal domains capable of sensing modifications in the ER environment and cytosolic domains that trigger signaling pathways, resulting in reduced protein synthesis and increased ER folding capacity to ultimately preserve ER functions and cell viability. In addition, proteins that fail to correctly fold can be deployed to the ER distal secretory pathway, the ER-associated protein degradation (ERAD) pathway of the UPR (Hwang and Qi, 2018). In this case, misfolded proteins are retro-translocated from the ER back to the cytosol for degradation. Finally, in case of persistent stress, the UPR can initiate cell death programs.

Autophagy is another highly conserved process in which eukaryotic cells rely on to maintain cell homeostasis - in this case, by degrading and recycling cytoplasmic components, such as defective organelles or protein aggregates (Mizushima et al., 2008; Klionsky et al., 2014; Siqueira et al., 2018). The autophagosomes display a characteristic double-membrane structure that sequesters cytosolic targets, known as cargos, for degradation. Autophagy is carried out by at least 30 conserved ATG proteins divided into macromolecular complexes. Other hundreds of proteins have been shown to modulate this process in different contexts, including the master homeostatic regulator mechanistic target of rapamycin (mTOR). The formation of autophagosomes is divided into three successive stages: (i) initiation, which involves the ULK1-ATG13-FIP200 complex; (ii) membrane nucleation, that is dependent on the Beclin 1 (BECN1)-ATG14-PI3K complex, and (iii) membrane elongation that requires ATG8/LC3 lipidation. LC3 lipidation is a hallmark of autophagy and is established by a covalent linkage of cytosolic LC3 to the lipid phosphatidylethanolamine on the surface of the autophagosome, which enables autophagosome elongation and recruitment of autophagy targets (Reggiori and Klionsky, 2005; Carneiro and Travassos, 2013). The entire process, which has been named autophagic flux, is completed when autophagosomes fuse with lysosomes and the cargo is degraded in autophagolysosomes.

There are many parallels between ISR/UPR and mTOR/ autophagy pathways, as they are both highly conserved signaling modules that regulate essential metabolic circuits, both in homeostatic and stress conditions. Also, the ER and autophagosomes are intrinsically linked since the former seems to be an essential source of lipids for the formation of the latter, and also provides a site for ATG14 anchoring, which plays a crucial role in recruiting the BECN1-ATG14-PI3K complex from the cytosol to sites of autophagosome initiation in response to starvation (Diao et al., 2015; Tan et al., 2016). Besides, localized phosphorylation of lipids by PI3K generates ER domains called omegasomes enriched in phosphatidylinositol-3-phosphate (Ptdlns3P) that recruit Ptdlns3P-effector proteins to generate sites for autophagosome nucleation and expansion (Mercer et al., 2018). In the context of ER stress, autophagy can be accessory by participating in the degradation of protein aggregates through ERAD (II), which is an alternative to the classic ubiquitinproteasome ERAD, designated ERAD (I), that clears most soluble misfolded proteins (Rashid et al., 2015).

Both UPR and autophagy are often observed during viral infections, in some cases as a host cell defense mechanism limiting viral replication and, in others, contributing to viral 
replication and establishment of infection (Blázquez et al., 2014; Chan, 2014; Xiao and Cai, 2020). Viral infections are a major cause of cellular stress as viruses are able to manipulate several cellular processes to complete their replicative cycle (RíosOcampo et al., 2018). In particular, the ability to divert the cell protein translation machinery to produce massive amounts of viral proteins profoundly impacts ER physiology (Gale et al., 2000). Furthermore, plus-stranded RNA viruses can pose an additional challenge for ER homeostasis as they synthesize their genome in association with extensive virus-induced rearrangements of intracellular membranes - including ER, endosomes or mitochondria (Romero-Brey and Bartenschlager, 2014). Included in this group are many viruses that cause disease in animals and humans, such as flaviviruses (for example, Dengue virus, Zika virus, Yellow fever virus, and West Nile virus), alphaviruses (for example, Chikungunya virus and Mayaro virus), and coronaviruses (for example, SARS-CoV, MERS-CoV, and SARS-CoV-2) (Jheng et al., 2014; Lee et al., 2018; Mehrbod et al., 2019). In the following sections of this review, we will discuss how UPR signaling and autophagy intersect in homeostatic conditions and in circumstances of mild and severe cell stress. In the final sections, we will take this discussion to the context of coronaviruses infections and highlight how these stress response pathways interfere with their replicative cycle.

\section{UPR AND AUTOPHAGY IN HOMEOSTATIC AND STRESS CONDITIONS}

Initially, PERK, IRE1, and ATF6 were considered to be active only when protein misfolding was detected in the cell. However, the identification of novel binding partners for these proteins suggest they participate in protein complexes that are important for regulating mitochondrial bioenergetics, cytoskeleton dynamics and membrane contacts (Hetz and Papa, 2018). For example, both PERK and IRE1 are present in mitochondria-associated membranes (MAMs) and the latter mediates the transfer of $\mathrm{Ca}^{2+}$ from the ER to mitochondria, thus regulating mitochondrial bioenergetics and physiology (MAMs) (Vliet et al., 2017; Urra et al., 2018; CarrerasSureda et al., 2019). The absence of IRE1 in MAMs led to AMPactivated protein kinase (AMPK) phosphorylation and activation of autophagy, as determined by increased basal levels of LC3B, a key component of the autophagosomal membrane (Carreras-Sureda et al., 2019). PERK and IRE1 also directly interact with components of the actin cytoskeleton. PERK regulates intracellular $\mathrm{Ca}^{2+}$ fluxes by forming dimers upon detection of increased cytosolic $\mathrm{Ca}^{2+}$ levels and then interacting with filamin A to reorganize the cytoskeleton and contact sites between the ER and the plasma membrane (Vliet et al., 2017). Likewise, the dimerization of IRE1 promotes its interaction with filamin A and controls cell migration (Urra et al., 2018). Together, these recent studies show that, in addition to their role in UPR, dimerization of PERK and IRE1 also participate in cell homeostatic pathways unrelated to ER stress.

In conditions where misfolded proteins accumulate in the ER lumen, chaperone binding immunoglobulin protein $(\mathrm{BiP} /$ GRP78) is recruited and thus detached from the luminal domains of the three ER sensors to which it was bound, releasing PERK and IRE1 from their inactive monomeric states and allowing ATF6 to transit to the Golgi (Smith and Wilkinson, 2017) (Figure 1). Activation of the UPR triggers two distinct events to mitigate protein misfolding: a quick reaction that involves phosphorylation of targets to immediately reduce protein synthesis and increase protein degradation; and a more durable response consisting of transcriptional upregulation of hundreds of target genes to restore proteostasis (Hetz and Papa, 2018).

An instantaneous reaction to ER stress is initiated by dimerized PERK, which phosphorylates eIF2 $\alpha$ in the cytosol leading to global attenuation of protein synthesis by forming an inhibitory complex with eIF2B that restricts its ability to bind to Met-tRNA initiator (Adomavicius et al., 2019). Paradoxically, at the same time, it initiates the translation of specific mRNAs that contain internal ribosomal entry sites. These include ATF4, which induces genes involved in redox homeostasis, amino acid metabolism, protein synthesis, apoptosis, and autophagy (Hetz et al., 2020). Under hypoxia, ATF4 promotes upregulation of LC3B through direct binding to a cyclic AMP response element-binding site in the $L C 3 B$ promoter (Rzymski et al., 2010). This process likely has a protective role for the cell, as inhibition of autophagy, under those circumstances, led to metabolic consequences of hypoxic stress (Rzymski et al., 2010). ATF4 also induces the expression of CHOP and, together, they transcriptionally activate genes involved in autophagy. These include genes encoding proteins involved in the formation and maturation of the autophagosome, such as Becn 1 (which encodes BECN1) and genes from the ubiquitin-like protein (Ubl) system, like Atg12 and Map1-lc3b (for LC3B) (B'chir et al., 2013). Genes encoding the activating enzyme (Atg7), the target of ATG12 attachment (Atg5), as well as genes encoding cargo receptors that are involved in specific degradation of ubiquitinated substrates, like $p 62$, are also upregulated by the eIF2 $\alpha / \mathrm{ATF} 4 / \mathrm{CHOP}$ branch (B'chir et al., 2013). Finally, protein synthesis is restored when eIF $2 \alpha$ is dephosphorylated by protein phosphatase 1 (PP1) regulatory subunit GADD34, which is also induced by ATF4 when ER stress is resolved (Hetz et al., 2020).

ER stress also results in dimerization of the transmembrane protein kinase/endoribonuclease IRE1, which autophosphorylates to switch on its RNase activity that consists of excising a short 26nucleotide intron from the mRNA encoding transcription factor XBP1 (Hetz et al., 2020). This processing generates the spliced Xbp1 mRNA, which is ultimately translated into the transcription factor XBP1s that upregulates genes involved in ER protein translocation, folding and secretion, as well as degradation of misfolded proteins mainly by ERAD (Hetz et al., 2020). IRE1 also induces a process known as regulated IRE1-dependent decay (RIDD), in which IRE1 cleaves and leads to the degradation of a small set of mRNAs or miRNAs (Hetz et al., 2020) (Figure 1). IRE1 activates autophagy in a more indirect manner, by interacting with adapter proteins like tumor necrosis factor (TNF) receptor- associated factor 2 (TRAF2) and apoptosis signal-regulating kinase 1 (ASK1), forming the IRE1/TRAF2/ 


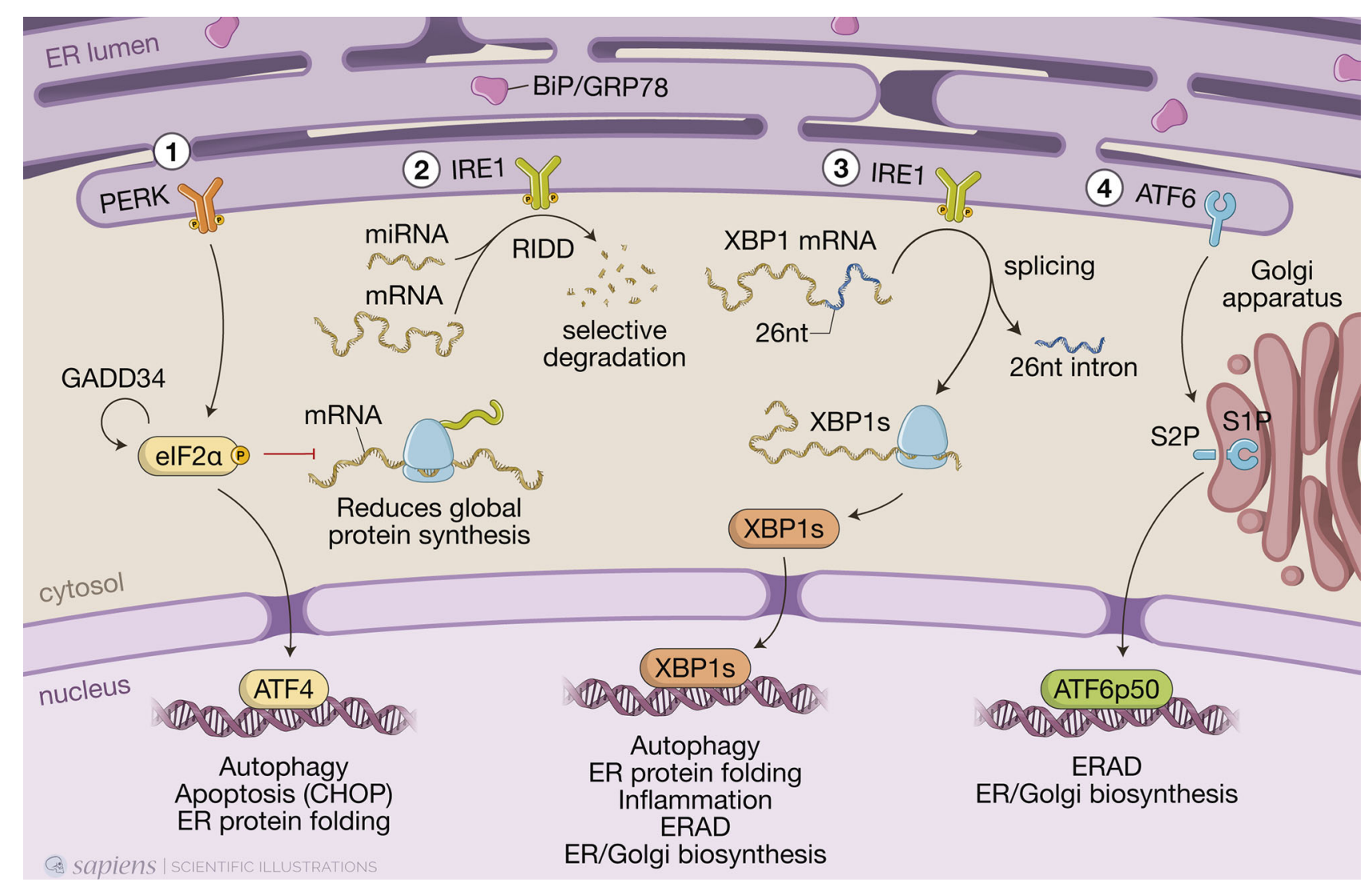

FIGURE 1 | The three branches of the unfolded protein response (UPR). When misfolded proteins accumulate in the endoplasmic reticulum (ER) lumen, chaperone binding immunoglobulin protein (BiP/GRP78) is detached from the luminal domains of the three ER sensors to which it was bound, allowing PKR-like ER protein kinase (PERK) and inositol-requiring protein-1 (IRE1) to form homodimers and activating transcriptional factor-6 (ATF6) to transit to the Golgi. (1) PERK phosphorylates the $\alpha$-subunit of the eukaryotic translation initiation factor 2 (elF2 $\alpha$ ), resulting in a global reduction of protein synthesis while still maintaining translation of a few key proteins, such as activating transcription factor 4 (ATF4), which induces expression of genes involved in redox homeostasis, amino acid metabolism, protein synthesis, autophagy and apoptosis, such as the transcription factor C/EBP homologous protein (CHOP). Protein synthesis is restored when elF2 $\alpha$ is dephosphorylated by protein phosphatase 1 (PP1) regulatory subunit GADD34, which is also induced by ATF4 when ER stress is resolved. (2) IRE1 autophosphorylates to switch on its RNase activity, inducing a process known as regulated IRE1-dependent decay (RIDD), in which IRE1 cleaves and leads to the selective degradation of a small set of mRNAs or miRNAs. (3) IRE1 also excises a short 26-nucleotide intron from the mRNA encoding transcription factor X-boxbinding protein 1 (XBP1), generating the spliced Xbp1 mRNA, which is ultimately translated into the transcription factor XBP1s that, like ATF4, upregulates genes involved in multiple cell signaling pathways, such as ER-associated protein degradation (ERAD). (4) Full length ATF6 translocates from the ER to the Golgi, where it is cleaved by site-1 protease (S1P) and site-2 protease (S2P). This releases a cytosolic fragment which then transits to the nucleus, transcription factor ATF6p50, which drives a transcriptional program to reestablish homeostasis.

ASK1 complex that activates c-Jun N-terminal kinase (JNK). JNK phosphorylates transcription factor c-Jun, which induces expression of Becn1 (Senft and Ronai, 2015; Liu et al., 2020). Although activation of JNK by IRE1 promotes, at first, this protective autophagic pathway, during prolonged ER stress, it can turn into autophagy-dependent cell death (Liu et al., 2020; Lindner et al., 2020).

Upon sensing ER stress, full-length ATF6 (ATF6p90) translocates from the ER to the Golgi apparatus, where it is cleaved by site-1 protease (S1P) and site-2 protease (S2P) and releases a cytosolic fragment containing a basic leucine zipper (bZIP) transcription factor, ATF6p50, which then transits to the nucleus (Hetz et al., 2020) (Figure 1). ATF6p50 and XBP1s act simultaneously and may form heterodimers, driving specific gene expression programs that bring about chaperone activation and ER/Golgi biogenesis to increase the cell secretory capacity (Shoulders et al., 2013; Hetz et al., 2020).

As we have mentioned above, the type, intensity, and duration of circumstances that induce ER stress are critical in determining the cell fate upon UPR activation. Initially, the UPR attempts to resolve the misfolded protein build-up, but persistent and unresolved ER stress is bound to cause cell death by inducing apoptosis (Yao et al., 2020). For example, the different outcomes resulting from ATF4 activation make PERK and phosphorylated eIF2 $\alpha$ crucial for determining the cell fate (Liu et al., 2020). Regarding the XBP-1 branch, while activation of Xbp1 mRNA splicing is transient and attenuated after prolonged stimulation, the activity of RIDD can be sustained over time and eventually contribute to cell death (Hetz and Papa, 2018). It is important to note that although a variety of mechanisms by which UPR 
promotes apoptosis have been described, each UPR pathway contribution to this outcome is modest, suggesting the existence of cell-type specific networks that arbitrate the cell fate under severe ER stress. The mechanisms by which UPR regulates the balance between cell survival and apoptosis have been extensively reviewed elsewhere (Hetz and Papa, 2018; Hetz et al., 2020).

\section{UPR, AUTOPHAGY AND COV INFECTIONS}

Coronaviruses ( $\mathrm{CoV}$ ) belong to the Coronaviridae family, which together with Roniviridae and Arteviridae form the order Nidovirales (Fung and Liu, 2019a). CoV infects an extensive range of birds and mammals, with several of them being economically important pathogens, including the avian infectious bronchitis virus (IBV) that causes severe respiratory and kidney diseases in poultry; the bovine coronavirus (BCoV) that causes respiratory tract diseases and diarrhea in cattle; feline infectious peritonitis virus (FIPV) that causes a fatal systemic disease in cats; and the transmissible gastroenteritis virus (TGEV) that causes diarrhea in pigs (Domańska-Blicharz et al., 2020). In humans, CoV are responsible for up to $30 \%$ of colds (Mesel-Lemoine et al., 2012). Importantly, CoVs have repeatedly demonstrated the ability to cross the species barrier and jump from non-human hosts to humans in a process known as zoonosis (Ye et al., 2020). In 2003, in the Chinese province of Guangdong, SARS-CoV-2 emerged as the etiological agent of the newly described severe acute respiratory syndrome (SARS), with high mortality rate. It was suggested that SARS-CoV was originated from bats and likely jumped to humans via some intermediate host (probably, palm civets) (Cheng et al., 2007). Nine years later, another zoonosis, this time originating from dromedaries, was detected in Saudi Arabia and named MiddleEast respiratory syndrome (MERS), caused by MERS-CoV (Mohd et al., 2016). In neither case, the fear of a pandemic was confirmed. More recently, however, another SARS-inducing $\mathrm{CoV}$ - now named SARS-CoV-2 - emerged in Wuhan (China) to cause a pandemic that, to date, has infected 135 million people and killed more than 2,92 million people around the world (https://github.com/CSSEGISandData/COVID-19).

\section{COV REPLICATION: ER, AUTOPHAGY AND THE ORIGINS OF DMVs}

CoVs are enveloped positive-sense RNA viruses (Chen et al., 2020). The first two-thirds of the genome consists of 2 large overlapping open reading frames, which encode 16 nonstructural proteins (NSPs), including proteases, RNAdependent RNA polymerase (prRdRp), RNA helicase, primase, and others, that form the viral replication and transcription complexes (RTCs), a platform to propagate viral mRNAs. The remaining portion of the genome includes interspersed open reading frames for the structural proteins - envelope (E), membrane $(\mathrm{M})$, nucleocapsid $(\mathrm{N})$, and the highly glycosylated spike (S) protein that projects from the viral envelope - as well as several accessory proteins generally nonessential for replication in tissue culture but capable of suppressing immune responses and enhancing pathogenesis (Ulferts et al., 2009). Infection begins when the viral $S$ protein attaches to its complementary host receptor, angiotensin I converting enzyme 2 (ACE2) in the case of SARS-CoV-2, allowing the virus to enter the host cells by endocytosis or direct fusion of the viral envelope with the host membrane (Hoffmann et al., 2020). Once inside the cell, the virus induces massive rearrangement of the intracellular membrane network to generate double-membrane vesicles (DMVs) (Prentice et al., 2003). CoV then targets their RTCs on the DMV-limiting membranes through multi-spanning transmembrane proteins (NSP3, NSP4, and NSP6) (Reggiori et al., 2010). The subgenomic viral RNAs are translated into structural and accessory proteins - transmembrane structural proteins (S, M, and E) are synthesized, inserted, and folded in the ER and transported to the ER-Golgi intermediate compartment (ERGIC), which is a structural and functional continuance of the $\mathrm{ER}$, whereas $\mathrm{N}$ proteins are translated in the cytoplasm (Senger et al., 2020). Virion assembly occurs in the ERGIC, and particles are exported through a secretory pathway in smooth-wall vesicles, which ultimately fuse with the plasma membrane to release the mature virus particle (Fehr and Perlman, 2015).

Early on, a role for autophagy in RNA virus replication has been an attractive hypothesis because of its association with complex membrane rearrangements in the cytoplasm that can generate opposed double membranes. Indeed, DMVs resemble autophagosomes and are seen in large numbers in the cytosol of $\mathrm{CoV}$-infected cells. In addition to DMVs, CoV replication complexes share other features of autophagosomes such as colocalization with multiple organelle markers and the acquisition of lysosomal markers throughout infection (Prentice et al., 2003). There have been different perspectives on the origin of $\mathrm{CoV}$ induced DMVs - late endosomes, autophagosomes, and early secretory pathways have all been implicated as the membrane source of DMVs (Chen et al., 2020; V'kovski et al., 2020; Wolff et al., 2020). The major difficulty in solving this issue has been the lack or undetectable levels of marker proteins of subcellular organelles (Reggiori et al., 2011). Accumulating data now indicate that ER-derived membranes are the major source for DMVs formation: (i) several viral proteins, including proteins that are part of the RTCs such as NSP3 and NSP4, are glycosylated in the ER; (ii) also ectopically expressed NSP4 is found in the ER and moves to DMVs upon viral infection; (iii) blocking early steps of the secretory pathway abolishes CoV replication; (iv) electron tomography of cells infected with either SARS-CoV or mouse hepatitis virus (MHV) showed that DMVs are part of a reticulovesicular network of modified ER membranes with double-stranded RNA (dsRNA) inside; and, (v) the subunit of the ER translocon, $\operatorname{Sec} 1 \alpha$, is found on rearranged membranes during SARS-CoV infection (Knoops et al., 2008; Angelini et al., 2013). Finally, as we will discuss below, recent studies have suggested that DMVs biogenesis might be linked to ERAD tuning pathway. More recently, an effort to establish a compendium of host factors required for $\mathrm{CoV}$ 
infection, including SARS-CoV-2 and other seasonal CoV, identified an absolute requirement for the TMEM41B for infection with all the $\mathrm{CoV}$ tested. TMEM41B is a ER-transmembrane protein involved in autophagy, which, again, argues in favor of a role of the ER and components of the autophagy pathway in DMVs biogenesis (Schneider et al., 2021). Thus, CoV replication is structurally and functionally linked to the ER and autophagy.

\section{AUTOPHAGY: IS IT REQUIRED OR A CONCOMITANT EVENT WITH COV REPLICATION?}

Accumulated evidence has shown that CoVs interact differentially with components of the autophagic pathway with potential for both utilization of its components for replication and attenuation of autophagy but full understanding of their link to autophagy still awaits further investigation.

$\mathrm{MHV}$ is considered a prototype $\mathrm{CoV}$ and has been extensively used to investigate mechanistic details of the replication and assembly of $\mathrm{CoV}$ in vitro and in vivo. Early studies have shown that MHV induced the formation of DMVs derived from autophagosomes and that proteins known to localize to replication complexes (p22 and N) colocalize with $\mathrm{LC}^{+}$foci in infected cells throughout the entire course of infection. MHVinduced autophagy was impaired in murine embryonic stem cells lacking ATG5, resulting in decreased viral yield (Prentice et al., 2003). In addition, atg5 knockout cells displayed a deranged morphology of the membranes, particularly hyper-swollen RER containing multiple vesicles, but no autophagosome formation suggesting that the RER might be the source of membranes for replication complexes. The reconstitution of the atg5 knockout with an expression plasmid restored viral yields implying the autophagy is required for viral replication (Prentice et al., 2003). Even though the molecular mechanisms were not determined, the authors hypothesized that the formation of DMVs could serve to sequester and concentrate viral replicases that were translated in the ER and that MHV may have evolved to utilize a preexisting cellular process - autophagy - to maximize replication efficiency. In contrast, Zhao et al. (2007) showed that in bonemarrow derived macrophages (BMMs) or primary mouse embryonic fibroblasts (MEFs) neither ATG5 nor an intact autophagic flux were required for $\mathrm{MHV}$ replication or release (Zhao et al., 2007). Considering viruses tropism to different cell types and the different permissiveness for viral replication among these cell types, it is possible that these discrepancies could be, at least in part, related to the different experimental models used (Figure 2).

Following studies suggested that $\mathrm{CoV}$ might not require an intact autophagic flux to replicate but still exploit components of the autophagic pathway to enhance infection. In this sense, another gene essential for autophagy, atg7, was shown to be dispensable for the formation of MHV-induced DMVs and viral replication in MEFs (Reggiori et al., 2010). Nevertheless, it was observed that endogenous nonlipidated LC3 extensively colocalized with the DMV protein markers, NSP2 and NSP3, and that downregulation of LC3, but not inactivation of host cell autophagy, protected cells from $\mathrm{CoV}$ infection as a result of defects in DMVs biogenesis. Of note, ectopically expressed GFPLC3, which is widely used to track autophagosomes, colocalized with neither NSP2 nor NSP3 but ectopically expressed Cterminally HA-tagged, nonlipidable LC3 did (Reggiori et al., 2010). This observation distinguishes DMVs from autophagosomes as LC3 lipidation, which is indispensable for autophagosome elongation, is not required for its association with DMVs. This feature is reminiscent of ERAD tuning vesicles known as EDEMosomes. Post-translational regulation of ERAD factors contained in the ER lumen by rapid and selective removal, which is critical for ER homeostasis, is known as ERAD tuning. In addition to chaperones and folding enzymes, ER also contains ERAD factors that recognize non-native proteins, extract them from the folding machinery and ensure their transport for proteasomal degradation. The ERAD regulators EDEM1, OS-9, and others are removed from the ER in vesicles that display LC3-I in their limiting membrane - the EDEMosomes - and degraded by endo-lysosomal enzymes. Based on this, the authors propose a mechanism by which MHV hijacks the ERAD tuning pathway to coopt cellular membranes for DMV formation and support viral RTCs. Indeed, MHV infection caused accumulation of EDEM1 and OS-9 in the DMVs, and this was independent of autophagy as atg7 deletion did not affect the intracellular levels of EDEM1 as it did those of $\mathrm{p} 62$, an autophagy substrate. Importantly, viralcoopting of this cellular process blocks the normal clearance of these vesicles as illustrated by data showing that, in non-infected cells, EDEM1 has a half-life of about 1 hour but upon MHV infection, is still found after several hours of infection even with virus-induced host translational shutoff, indicating that there is actually defective clearance of EDEM1 in infected cells.

A screen using individual IBV non-structural proteins for their ability to induce autophagy showed that NSP6 was located to the ER and induced ER puncta containing DFCP1 and ATG5 (Cottam et al., 2011). NSP-6-induced autophagosomes required ATG5 and the recruitment of lipidated LC3-II, features of classic autophagosomes generated in the ER via omegasomes rather than EDEMosomes. Furthermore, class 3 PI3 kinase activity was also required, indicating PtdIns(3)P are generated from ER lipids to build phagophores. In the IBV model, the infection or ectopic expression of NSP6 induces a complete autophagic flux as the autophagosomes are fused with Lamp1-positive vesicles, which show their ability to deliver the cargo to the lysosomes. However, an intact autophagy flux is not required for viral replication as this was unaffected by pharmacological autophagy inhibitors or silencing of ATG5. NSP6 orthologs from other CoV (SARS-CoV and $\mathrm{MHV}$ ) also localized to the ER, from where they generate autophagosomes via an omegasome intermediate. Targeting NSP6 to the ER resulted in partial XBP1 splicing and undetectable increase in $\mathrm{CHOP}$ expression, indicating that ER stress is limited and not mechanistically involved in autophagy induction (Cottam et al., 2014). It would be interesting to investigate other branches of the UPR since, as we mentioned 


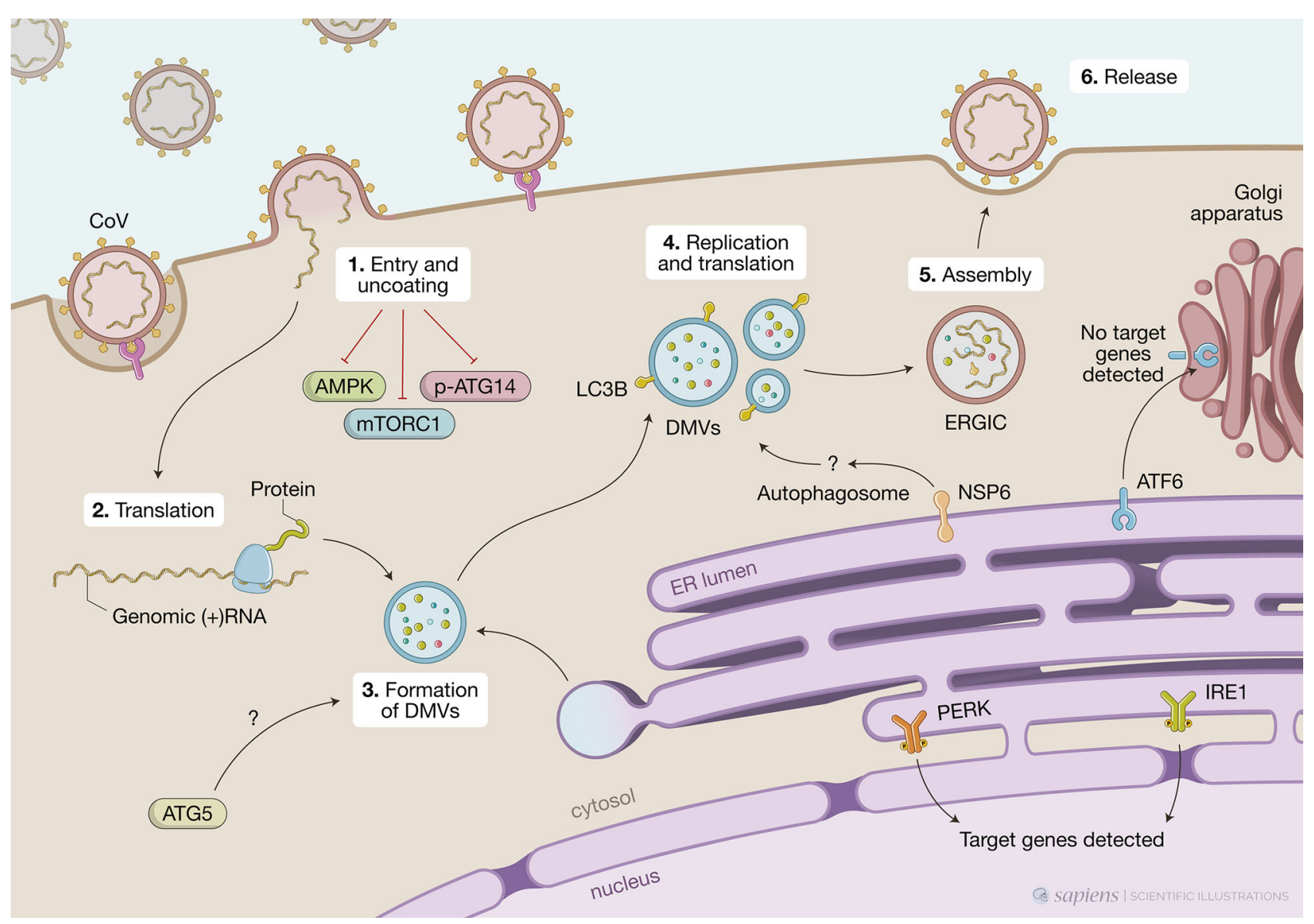

FIGURE 2 | Unfolded protein response (UPR), autophagy and coronavirus (CoV) infections. This figure represents data obtained by using different cell models infected by CoV viruses mouse hepatites virus (MHV), infectious bronchitis virus (IBV), SARS-CoV or SARS-CoV-2. CoV infection begins when the viral S protein attaches to its complementary host receptor, allowing the virus to enter the host cells by endocytosis or direct fusion of the viral envelop with the host membrane. The process of SARS-CoV2 virus entry and uncoating (1) downregulates AMPK and reduces phosphorylation of ATG14, disrupting the autophagy flux, while simultaneously inhibiting mTORC1, which promotes autophagy. Once inside the cell, the viral positive-sense RNA is translated (2) and the virus induces massive rearrangement of the intracellular membrane network to generate double membrane vesicles (DMVs) (3). CoV-induced DMVs may originate from late endosomes, autophagosomes or vesicles from the early secretory pathway. MHV-induced autophagy was impaired in cells lacking ATG5, which displayed a deranged morphology of the membranes and decreased viral yield. Nonlipidated LC3 extensively colocalizes with DMV protein markers and downregulation of LC3 protects cells from CoV infection as result of defects in DMVs biogenesis. CoV replication occurs within DMVs and transmembrane structural proteins (S, M and E) are synthesized in the ER (4). Non-structural protein NSP6 from IBV, MHV and SARS-CoV are localized in the ER and participate in autophagosome formation via omegasomes, which could be used for DMV formation. Finally, new viral particles are transported to the ER-Golgi intermediate compartment (ERGIC) for assembly (5) and exported through secretory pathway in smooth-wall vesicles, which ultimately fuse with the plasma membrane to release the mature virus (6). A high demand for membranes for DMV formation and virion exocytosis contributes to ER stress. MHV activates PERK/elF2 $\alpha$ and IRE1 and target genes from these two branches are detected upon infection. Cleavage of ATF6 can also be observed but no target genes are detected subsequently.

above, individual pathways might not represent the whole picture.

The same group investigated the characteristics of the autophagosomes induced by ectopic expression of NSP6 from several CoV (SARS-CoV, MHV or IBV) as well as IBV infection (Cottam et al., 2014). All of them induced the formation of a significant number of autophagosomes that were significantly smaller $(<0.5 \mu \mathrm{m}$ diameter $)$ than the ones induced by starvation $(>1.0 \mu \mathrm{m})$. Even with concomitant signals for autophagy activation, such as starvation or mTOR inhibition, IBV infection or IBV NSP6 ectopic expression limited expansion of omegasomes, thereby limiting the expansion of autophagosomes.
The authors showed that mTOR was missing from the surface of lysosomes of cells expressing NSP6 even when cultured in nutrient media and suggested that NSP6 prevents mTOR association with lysosomes limiting the formation of large autophagosolysosomes. It still to be addressed if NSP6 acts by directly interacting with other proteins at the surface of lysosomes or indirectly by affecting other signaling pathways. Although smaller, NSP6-induced autophagosomes could still take up SQSTM1/p62 and deliver it to lysosomes. Additional work will be necessary to understand if limiting autophagosome expansion brings any advantage for $\mathrm{CoV}$ replication. Given that the size of DMVs is much smaller than what is observed in starvation-induced autophagosomes, the authors 
hypothesize that these smaller autophagosomes could be used to generate DMVs (Cottam et al., 2014).

In contrast to the studies above that suggest a positive correlation between the induction of autophagy and $\mathrm{CoV}$ replication, other studies have proposed an inhibitory effect of $\mathrm{CoV}$ on the autophagic process. For instance, MERS-CoV seems to establish a tug of war in which at the same time that autophagy limits viral propagation, the virus is able to impair autophagic flux by activation the E3-ligase S-phase kinase-associates protein 2 (SKP2) (Gassen et al., 2019). SPK2 acts via FKBP5, a stressregulated protein involved in numerous pathways through scaffolding regulatory protein interactions and the involvement of AKT1 and PHLPP. Activated phosphorylated SPK2 polyubiquitinates the critical autophagy initiating protein BECN1 for proteasomal degradation. Conversely, pharmacological inhibition of SPK2 stabilizes BECN1, enhances autophagy, and restricts MERS-CoV propagation. Altogether these results indicate that autophagy is a relevant anti-MERS-CoV mechanism. Indeed, knocking down ATG5 resulted in a 52-fold increase in the formation of MERS-CoV particles (Gassen et al., 2019). More recently, the same authors have shown that similar to MERS-CoV, SARS-CoV-2 strongly reduced the autophagic flux in two different cell lines - the human bronchial epithelial cells NCI-H1299 and Vero cells (Gassen et al., 2020). This later study showed that the phosphorylated active forms of AMPK, AMPK substrates (LXRXX), AMPH downstream targets (TSC2 and ULK1) and mTORC1 were all downregulated upon SARS-CoV-2 infection. Concomitantly, increased levels of phosphorylated AKT1 were observed, leading to SKP2 activation and decreased BECN1 levels. This resulted in reduced ATG14 phosphorylation and oligomerization and subsequent lack of fusion of autophagosomes and lysosomes and disrupted autophagy flux. It had been previously suggested that the membrane-associated papain-like protease PLP2 (PLP2-TM) of SARS-CoV or MERS-CoV could be involved in blockage of autophagosomes-lysosomes fusion and suppression of the autophagic flux (Yang and Shen, 2020).

\section{ER STRESS INDUCES DIFFERENT SIGNALING CIRCUITS IN RESPONSE TO COV INFECTION -}

Despite the lack of consensus on the biogenesis of DMVs, it is clear that its membranes originate from the ER, whether it is through omegasomes/autophagosomes, EDEMosomes or both. Such high demand for membranes represents an additional burden to the ER that is also coopted to produce viral proteins. Indeed, during $\mathrm{CoV}$ replication, massive amounts of structural proteins are synthesized in the ER, and the production, folding and modification (in particular, extensive glycosylation of $\mathrm{S}$ protein) of these proteins increase the workload of the ER, eventually overloading its folding capacities and leading to UPR activation. Continuous depletion of the ER lipid content due to virion exocytosis also contributes to ER stress. Several pieces of evidence indicate that $\mathrm{CoV}$ infection induces ER stress: (i) genes and proteins involved in ER stress were shown to be upregulated in cells infected with SARS-CoV. Different studies showed that both the glucose-regulated protein 94 (GRP94) and GRP78/BiP are induced upon SARS-CoV infection in cell culture systems (Chan et al., 2006; Palmeira et al., 2020). In addition, infection with either MHV or SARS-CoV also results in upregulation of homocysteine-inducible, ER-stress inducible, ubiquitin-like domain member 1 (HERPUD1), an ER stress marker (Fung and Liu, 2014).

Among the three branches of UPR, the PERK-eIF2 $\alpha$ is the best characterized in $\mathrm{CoV}$-infected cells. For example, $\mathrm{MHV}$ induces significant eIF2 $\alpha$ phosphorylation and ATF4 upregulation in infected cells resulting in sustained translation repression. Even though there have been conflicting results in early studies on the role of the integrated stress response on $\mathrm{CoV}$ infections, it appears that both PERK and PKR, and subsequently eIF2 $\alpha$ phosphorylation, occur at the early stages of $\mathrm{CoV}$ infections in cell cultures. In cells infected with SARS-CoV, PERK, PKR and eIF2 $\alpha$ phosphorylation were detected as early as $8 \mathrm{~h}$ post-infection (Krähling et al., 2009). Even though it significantly inhibited SARS-CoV-induced apoptosis, the knock-down of PKR did not affect eIF2 $\alpha$ suggesting that this could be dependent on PERK activation. Supporting this is the previous observation that SARS-CoV accessory protein $3 \mathrm{a}$ induces PERK phosphorylation (Minakshi et al., 2009). Two complementary studies from the same group indicated that the PERK/PKR-eIF2 $\alpha$ pathway is also activated at early stages of IBV infection in vitro, leading to ERK1/2 phosphorylation and promoting cell survival. After $8 \mathrm{~h}$ of infection, however, eIF $2 \alpha$ is dephosphorylated as a feedback response due to the accumulation of GADD34, which is downstream of ATF4 and GADD153. Up-regulation of GADD153, which was partially blocked by silencing either PKR or PERK, also promoted apoptosis in IBV-infected cells (Wang et al., 2009; Liao et al., 2013). Altogether, these results highlight the delicate balance between cell survival and cell death upon UPR activation depending on the intensity and/or duration of stress.

The ORF3 protein of porcine epidemic diarrhea virus (PEDV), similar to SARS-CoV 3 a protein or human pathogenic coronavirus NL63 (hCoV-NL63) ORF protein, localizes to the ER and triggers ER stress by upregulating GRP78 and activating the PERK-eIF2 $\alpha$ pathway (Zou et al., 2019). This, in turn, results in induction of autophagy as shown by conversion of LC3-I into LC3-II. ORF3 protein is thought to function as an ion channel and to influence virus production and virulence (Wang et al., 2012).

Studies using MHV have demonstrated that the IRE1 axis also senses ER stress during infection, as shown by efficient splicing of $\mathrm{XBP} 1 \mathrm{mRNA}$ upon infection or overexpression of the $\mathrm{S}$ protein (Bechill et al., 2008). However, how this contributed to the cellular response was unclear since the protein product of the spliced XBP1 was not observed and genes known to be downstream of XBP1s - such as EDEM1, ER DNA J domaincontaining protein 4 (ERdj4), and protein kinase inhibitor of 58 $\mathrm{kDa}\left(\mathrm{p} 58^{\mathrm{IPK}}\right)$ - were not significantly up regulated following infection. It is possible that sustained eIF $2 \alpha$ phosphorylation and 
translational repression observed during $\mathrm{MHV}$ infection interfere with the translation of the XBP1 protein. Another possibility is that IRE1 operates through alternative signaling pathways. It has been demonstrated that activation of IRE1 was essential for autophagy induction upon infection with another CoV, IBV (Fung and Liu, 2019b). Autophagosome formation and autophagic flux were also dependent on ATG5 but independent of BECN1. In this model, XBP1 splicing was dispensable, and IRE1 signaled through ERK1/2 to modulate autophagy induction in infected cells and protect them from apoptosis. A more recent study demonstrated that IBV can induce significant splicing of XBP1 mRNA and subsequent upregulation of EDEM1, ERdj4 and $\mathrm{p} 58^{\mathrm{IPK}}$ in various cell lines (Fung et al., 2014). This was dependent on IRE1 activation as inhibiting or knocking down IRE1 effectively blocked IBVinduced XBP1 mRNA splicing and effector genes upregulation. In this context, the IRE1-XBP1 pathway seems to protect cells from apoptosis by modulating JNK and AKT phosphorylation. Altogether, these studies highlight how stress-related pathways intersect in different ways depending on cellular context and how different signaling circuits ultimately define cell fate. Also, it is important to evaluate the cell response as a whole, as it is not uncommon for RNA viruses to utilize only a subset of the components of stress response pathways that can signal through alternative pathways.

Similar to what was observed with IRE1, significant cleavage of ATF6 is observed following infection with MHV but the activation of target genes was not detected using luciferase reporter constructs under the control of ERSE promoters (Fung et al., 2014). In addition, ATF6 levels (full length and cleaved) significantly decrease at later time points of infection. The authors of this study suggest that global protein synthesis arrest following eIF $2 \alpha$ phosphorylation impedes ATF6 accumulation and subsequent activation of target genes (Bechill et al., 2008).

ATF6 was implicated in the response to SARS-CoV. On the 2003 SARS-CoV outbreak, the accessory protein $8 \mathrm{ab}$ was found in animals and early human isolates. This protein was found to co-immunoprecipitate with and induce ATF6 cleavage and nuclear translocation (Sung et al., 2009). At the peak of the epidemic, human isolates presented a 29-nt deletion in the middle of ORF8, resulting in two smaller ORFs that encode the truncated polypeptides ORF8a and ORF8b. More recently, it was demonstrated that ORF8b forms insoluble intracellular aggregates leading to ER stress, lysosomal damage, and subsequent activation of the master regulator of the autophagy and lysosome machinery, transcription factor EB (TFEB), leading to increased autophagic flux. ORF8b aggregates are partially degraded by the autophagy-lysosome pathway. Depending on the cell type this may result in either cell death (as observed in epithelial cells) or a robust NLRP3 inflammasome activation by directly targeting its LRR domain with the release of inflammatory mediators (as observed in macrophages). In wildtype macrophages, ORF8b induces NLRP3-dependent pyroptosis while in NLRP3-deficient macrophages, cell death results from mitochondrial dysfunction (Shi et al., 2019).

\section{MANIPULATION OF UPR AND AUTOPHAGY AS A STRATEGY TO LIMIT COV REPLICATION}

As the current SARS-CoV-2 pandemic progressed, the repurposing of drugs already approved for human use could represent the fastest way to limit viral spread and/or severe disease, save lives, and prevent the collapse of health care systems. Drugs known to modulate both UPR (e.g. thapsigargin) (Al-Beltagi et al., 2021) and autophagy (e.g. hydroxichloroquine) (Senger et al., 2020) were shown to be broad-spectrum inhibitors for several respiratory viruses, including $\mathrm{CoV}$, and emerged as candidates. Even though experimental findings have not yet been translated into efficient treatment and the use of these drugs remains controversial, the literature offers a rationale to target UPR and autophagy in the context of $\mathrm{CoV}$ infections. As we have described in this review, these two events are an integral part of the host cell-virus interactions and can be involved in different steps of the viral replicative cycle - from the establishment of replicative niches (DMVs) to regulating cell death. In particular, viral usage of double membranes vesicles resembling autophagosomes as a platform for replication, as a source of membrane for their envelope, as well as an intracellular shuttle for their exocytosis has been reported. Even though the precise mechanisms of DMVs biogenesis are still a matter of debate, components of the autophagic machinery and/or autophagic flux are often found to be necessary. Finally, all CoV identified so far have evolved to manipulate the autophagy pathway in some way, which once again argues that this pathway is essential for viral replication cycle.

One of the first proposed clinical trials in the wake of the COVID-19 pandemic draw huge worldwide attention to the putative benefits of hydroxychloroquine (HCQ) in the early treatment of patients infected with SARS-CoV-2 (Senger et al., 2020). Previously, HCQ had been shown to inhibit MERS-CoV replication in vitro in a screening of FDA-approved compound library (Touret et al., 2020). In experimental SARS-CoV infections, HCQ was able to limit inflammation markers even though it has not affected viral titers in the lungs of infected mice (Maisonnasse et al., 2020). The mechanisms proposed to explain the effects of HCQ on SARS-CoV-2 infection included the ability to interfere with ACE2 terminal glycosylation by raising the $\mathrm{pH}$ in the Golgi compartment thereby reducing the cell surface expression of SARS-CoV2 receptor (Kalra et al., 2020). In addition, by accumulating in the acidic organelles such as endosomes and lysosomes and neutralizing their $\mathrm{pH}$, it can also disrupt fusion of viral endosomes with lysosomes or the activity of proteases preventing the cleavage of $S$ protein blocking early steps in the viral life cycle (Chen and Geiger, 2020). Finally, HCQ is a wellknown inhibitor of autophagy flux by impairing autophogasome fusion with lysosomes (Mauthe et al., 2018). After months of intense debate worldwide and follow-up studies, the efficacy of HCQ to treat COVID-19 was not confirmed, and its use has been discouraged by the FDA because of the risk of side effects. Nevertheless, one should not disregard autophagy as a potential pharmaceutical target because (i) it is a critical host process that controls all steps harnessed by SARS-CoV-2 and (ii) a collection of 
other drugs known to be autophagy modulators were shown to reduce or block SARS-CoV-2 infection in vitro. For instance, work from Gorshkov et al. (2020) identified 6 compounds known to be autophagy modulators that were able to reduce the cytopathic effects of SARS-CoV-2 in vitro with $\mathrm{EC}_{50}$ values ranging from 2.0 to $13 \mu \mathrm{M}$ and selectivity indices ranging from 1.5 to $>10$-fold and their efficacy for inhibiting autophagy correlated with their ability to prevent SARS-CoV-2 cytopathic effects in various cell lines (Gorshkov et al., 2020).

As mentioned previously in this review, work from Gassen et al. (2019) showed that in their experimental conditions, MERS$\mathrm{CoV}$ and SARS-CoV-2 impair autophagy by targeting BCL-1 for proteasomal degradation upon activation of SKP2. Blocking SKP2 with different compounds, including FDA-approved drugs, stabilized BECN1 limiting MERS-CoV and SARS-CoV-2 propagation which, in this case, contrary to HCQ that inhibits autophagy, indicates that boosting autophagy could be an efficient anti-CoV mechanism (Gassen et al., 2019). These authors tested a collection of known autophagy modulators such as spermidine, spermine, rapamycin, AKT1 inhibitors such as MK-2206, and the BCN1 stabilizing antihelmintic drug niclosamide and further advanced the idea that inducing autophagy can limit SARS-CoV infections (Gassen et al., 2020).

Manipulating the UPR has also been shown to be a potential strategy to treat infections with respiratory viruses. Al-Beltagi et al. showed that, in vitro, non-cytotoxic levels of thapsigargin (TG), an inhibitor of the ER-Ca ATPase pump, can block the replication of relevant human respiratory viruses such as $\mathrm{CoV}$ (including SARS-CoV-2), Respiratory Syncytial Virus and Influenza (Al-Beltagi et al., 2021). The effects of TG on CoV (hCoV-229E, MERS-CoV and SARS-CoV-2) replication have also been reported in another recent study (Shaban et al., 2020). The authors had previously shown that, in the case of CoV OC43 and RSV, TG-induced ER stress leading to UPR was a central innate immune driver that mediated several downstream host antiviral mechanisms that are particularly effective in blocking the replication of different RNA viruses, which include increased expression of ER stress genes (DDIT3, HSPA5 and HSP90B1) and was accompanied by reduced viral transcription and viral protein expression (Goulding et al., 2020).

\section{CONCLUDING REMARKS}

As we understand cellular stress responses, it becomes clear that multiple pathways that are activated independently intersect in

\section{REFERENCES}

Adomavicius, T., Guaita, M., Zhou, Y., Jennings, M. D., Latif, Z., Roseman, A. M., et al. (2019). The Structural Basis of Translational Control by EIF2 Phosphorylation. Nat. Commun. 10, 2136. doi: 10.1038/s41467-019-10167-3

Al-Beltagi, S., Preda, C. A., Goulding, L. V., James, J., Pu, J., Skinner, P., et al. (2021). Thapsigargin is a Broad-Spectrum Inhibitor of Major Human Respiratory Viruses: Coronavirus, Respiratory Syncytial Virus and Influenza a Virus. Viruses 13, 234. doi: 10.3390/v13020234 different ways, resulting in specific signaling circuits tailored to the cellular context. In this sense, it has been recently shown that there is a crosstalk between UPR and autophagy even in homeostatic conditions and it can be differentially modulated upon mild and severe or long-lasting stress. In the context of infections, stress-response mechanisms play multiple roles, including maintenance of homeostasis, fine-tuning of the immune response, and, in some cases, a direct anti-infectious role. The UPR/ISR and autophagy are known to limit the replication of several viruses. On the other hand, many RNA viruses, including $\mathrm{CoV}$, take advantage of these responses to enhance their replication. There has been much debate on the biogenesis of CoV DMVs, which is a crucial step of the viral replicative cycle. Despite the lack of consensus, it is clear that its membranes originate from the ER, whether through omegasomes/autophagosomes, EDEMosomes or both. In addition, even though a complete autophagic flux seems to be dispensable, components of the autophagic machinery may be required in "alternative" pathways for CoV DMVs formation and replication. Similarly, ER stress induced upon $\mathrm{CoV}$ infection signals both through canonical UPR response as well as "alternative" signaling involving MAP kinases. There have been enough pieces added to the puzzle to establish a role for UPR and autophagy in CoV replication. However, the missing pieces will define the signaling circuits involved. This will be particularly important for the design of vaccines and therapeutic strategies to face the pandemic.

\section{AUTHOR CONTRIBUTIONS}

EP, JB, LT, and LC conceived and wrote the manuscript. LC revised the manuscript. All authors contributed to the article and approved the submitted version.

\section{FUNDING}

EP is supported by a fellowship from Coordenação de Aperfeiçoamento de Pessoal de Nivel Superior (CAPES). JB is supported by a studentship from Fundação Carlos Chagas Filho de Amparo à Pesquisa do Estado do Rio de Janeiro (FAPERJ). Research in the labs of LT and LC are funded by Conselho Nacional de Desenvolvimento Científico e Tecnológico (CNPq) and FAPERJ. Programming. Front. Immunol. 8, 1719. doi: 10.3389/fimmu.2017.01719

B'chir, W., Maurin, A.-C. C., Carraro, V., Averous, J., Jousse, C., Muranishi, Y., et al. (2013). The EIF2 $\alpha /$ Atf4 Pathway is Essential for Stress-Induced 
Autophagy Gene Expression. Nucleic Acids Res. 41, 7683-7699. doi: 10.1093/ nar/gkt563

Bechill, J., Chen, Z., Brewer, J. W., and Baker, S. C. (2008). Coronavirus Infection Modulates the Unfolded Protein Response and Mediates Sustained Translational Repression $\nabla$. J. Virol. 82, 4492-4501. doi: 10.1128/jvi.00017-08

Blázquez, A.-B. B., Escribano-Romero, E., Merino-Ramos, T., Saiz, J.-C. C., and Martín-Acebes, M. A. (2014). Stress Responses in Flavivirus-Infected Cells: Activation of Unfolded Protein Response and Autophagy. Front. Microbiol. 5, 266. doi: $10.3389 /$ fmicb. 2014.00266

Carneiro, L. A., and Travassos, L. H. (2013). The Interplay Between Nlrs and Autophagy in Immunity and Inflammation. Front. Immunol. 4, 361. doi: 10.3389/fimmu.2013.00361

Carreras-Sureda, A., Jaña, F., Urra, H., Durand, S., Mortenson, D. E., Sagredo, A., et al. (2019). Non-Canonical Function of IRE1 $\alpha$ Determines MitochondriaAssociated Endoplasmic Reticulum Composition to Control Calcium Transfer and Bioenergetics. Nat. Cell Biol. 21, 755-767. doi: 10.1038/s41556-019-0329-y

Chan, S.-W. (2014). The Unfolded Protein Response in Virus Infections. Front. Microbiol. 5, 518. doi: 10.3389/fmicb.2014.00518

Chang, H. H. Y., Pannunzio, N. R., Adachi, N., and Lieber, M. R. (2017). NonHomologous DNA End Joining and Alternative Pathways to Double-Strand Break Repair. Nat. Rev. Mol. Cell Bio 18, 495-506. doi: 10.1038/nrm.2017.48

Chan, C.-P., Siu, K.-L., Chin, K.-T., Yuen, K.-Y., Zheng, B., and Jin, D.-Y. (2006). Modulation of the Unfolded Protein Response by the Severe Acute Respiratory Syndrome Coronavirus Spike Protein. J. Virol. 80, 9279-9287. doi: 10.1128/ jvi.00659-06

Chen, X., and Geiger, J. D. (2020). Janus Sword Actions of Chloroquine and Hydroxychloroquine Against Covid-19. Cell Signal 73, 109706. doi: 10.1016/ j.cellsig.2020.109706

Cheng, V. C. C., Lau, S. K. P., Woo, P. C. Y., and Yuen, K. Y. (2007). Severe Acute Respiratory Syndrome Coronavirus as an Agent of Emerging and Reemerging Infection. Clin. Microbiol. Rev. 20, 660-694. doi: 10.1128/cmr.00023-07

Chen, Y., Liu, Q., and Guo, D. (2020). Emerging Coronaviruses: Genome Structure, Replication, and Pathogenesis. J. Med. Virol. 92, 418-423. doi: 10.1002/jmv.25681

Costa-Mattioli, M., and Walter, P. (2020). The Integrated Stress Response: From Mechanism to Disease. Sci. New York N. Y. 368, 1-11. doi: 10.1126/science.aat5314

Cottam, E. M., Maier, H. J., Manifava, M., Vaux, L. C., Chandra-Schoenfelder, P., Gerner, W., et al. (2011). Coronavirus Nsp6 Proteins Generate Autophagosomes From the Endoplasmic Reticulum Via an Omegasome Intermediate. Autophagy 7, 1335-1347. doi: 10.4161/auto.7.11.16642

Cottam, E. M., Whelband, M. C., and Wileman, T. (2014). Coronavirus Nsp6 Restricts Autophagosome Expansion. Autophagy 10, 1426-1441. doi: 10.4161/ auto.29309

Diao, J., Liu, R., Rong, Y., Zhao, M., Zhang, J., Lai, Y., et al. (2015). Atg14 Promotes Membrane Tethering and Fusion of Autophagosomes to Endolysosomes. Nature 520, 563-566. doi: 10.1038/nature14147

Domańska-Blicharz, K., Woźniakowski, G., Konopka, B., Niemczuk, K., Welz, M., Rola, J., Socha, W., et al. (2020). Animal Coronaviruses in the Light of COVID19. J. Vet. Res. 64, 333-345. doi: 10.2478/jvetres-2020-0050

Fehr, A. R., and Perlman, S. (2015). Coronaviruses, Methods and Protocols. Methods Mol. Biol. Clifton N J. 1282, 1-23. doi: 10.1007/978-1-4939-2438-7_1

Fung, T. S., Huang, M., and Liu, D. X. (2014). Coronavirus-Induced ER Stress Response and Its Involvement in Regulation of Coronavirus-Host Interactions. Virus Res. 194, 110-123. doi: 10.1016/j.virusres.2014.09.016

Fung, T. S., Liao, Y., and Liu, D. X. (2014). The Endoplasmic Reticulum Stress Sensor Ire1 $\alpha$ Protects Cells From Apoptosis Induced by the Coronavirus Infectious Bronchitis Virus. J. Virol. 88, 12752-12764. doi: 10.1128/jvi.02138-14

Fung, T. S., and Liu, D. X. (2014). Coronavirus Infection, Er Stress, Apoptosis and Innate Immunity. Front. Microbiol. 5, 296. doi: 10.3389/fmicb.2014.00296

Fung, T., and Liu, D. (2019a). Human Coronavirus: Host-Pathogen Interaction. Annu. Rev. Microbiol. 73, 529-557. doi: 10.1146/annurev-micro-020518-115759

Fung, T., and Liu, D. (2019b). The ER Stress Sensor IRE1 and MAP Kinase ERK Modulate Autophagy Induction in Cells Infected With Coronavirus Infectious Bronchitis Virus. Virology 533, 34-44. doi: 10.1016/j.virol.2019.05.002

Gale, M., Tan, S.-L., and Katze, M. G. (2000). Translational Control of Viral Gene Expression in Eukaryotes. Microbiol. Mol. Biol. R 64, 239-280. doi: 10.1128/ mmbr.64.2.239-280.2000

Galluzzi, L., Pedro, J., Levine, B., Green, D. R., and Kroemer, G. (2017). Pharmacological Modulation of Autophagy: Therapeutic Potential and
Persisting Obstacles. Nat. Rev. Drug Discov. 16, 487-511. doi: 10.1038/ nrd.2017.22

Galluzzi, L., Yamazaki, T., and Kroemer, G. (2018). Linking Cellular Stress Responses to Systemic Homeostasis. Nat. Rev. Mol. Cell Biol. 19, 731-745. doi: 10.1038/s41580-018-0068-0

García, M. A., Gil, J., Ventoso, I., Guerra, S., Domingo, E., Rivas, C., et al. (2006). Impact of Protein Kinase PKR in Cell Biology: From Antiviral to Antiproliferative Action. Microbiol. Mol. Biol. R 70, 1032-1060. doi: 10.1128/mmbr.00027-06

Gassen, N. C., Niemeyer, D., Muth, D., Corman, V. M., Martinelli, S., Gassen, A., et al. (2019). Skp2 Attenuates Autophagy Through Beclin1-Ubiquitination and Its Inhibition Reduces Mers-Coronavirus Infection. Nat. Commun. 10, 5770. doi: 10.1038/s41467-019-13659-4

Gassen, N. C., Papies, J., Bajaj, T., Dethloff, F., Emanuel, J., Weckmann, K., et al. (2020). Analysis of SARS-Cov-2-Controlled Autophagy Reveals Spermidine, MK-2206, and Niclosamide as Putative Antiviral Therapeutics. Biorxiv 2020, 4.15.997254. doi: 10.1101/2020.04.15.997254

Girardin, S. E., Cuziol, C., Philpott, D. J., and Arnoult, D. (2020). The eIF2 $\alpha$ kinase HRI in innate immunity, proteostasis and mitochondrial stress. FEBS J. doi: $10.1111 /$ febs. 15553

Gorshkov, K., Chen, C. Z., Bostwick, R., Rasmussen, L., Xu, M., Pradhan, M., et al. (2020). The SARS-Cov-2 Cytopathic Effect is Blocked With Autophagy Modulators. bioRxiv. doi: 10.1101/2020.05.16.091520

Goulding, L. V., Yang, J., Jiang, Z., Zhang, H., Lea, D., Emes, R. D., et al. (2020). Thapsigargin At non-Cytotoxic Levels Induces a Potent Host Antiviral Response That Blocks Influenza a Virus Replication. Viruses 12, 1093. doi: $10.3390 / \mathrm{v} 12101093$

Hetz, C. (2012). The Unfolded Protein Response: Controlling Cell Fate Decisions Under ER Stress and Beyond. Nat. Rev. Mol. Cell Bio 13, 89-102. doi: 10.1038/ nrm 3270

Hetz, C., and Papa, F. R. (2018). The Unfolded Protein Response and Cell Fate Control. Mol. Cell 69, 169-181. doi: 10.1016/j.molcel.2017.06.017

Hetz, C., Zhang, K., and Kaufman, R. J. (2020). Mechanisms, Regulation and Functions of the Unfolded Protein Response. Nat. Rev. Mol. Cell Biol. 21, 421438. doi: 10.1038/s41580-020-0250-Z

Hoffmann, M., Kleine-Weber, H., Schroeder, S., Krüger, N., Herrler, T., Erichsen, S., et al. (2020). Sars-Cov-2 Cell Entry Depends on ACE2 and TMPRSS2 and is Blocked by a Clinically Proven Protease Inhibitor. Cell 181, 271-280.e8. doi: 10.1016/j.cell.2020.02.052

Hwang, J., and Qi, L. (2018). Quality Control in the Endoplasmic Reticulum: Crosstalk Between ERAD and UPR Pathways. Trends Biochem. Sci. 43, 593605. doi: 10.1016/j.tibs.2018.06.005

Jackson, S. P., and Bartek, J. (2009). The DNA-Damage Response in Human Biology and Disease. Nature 461, 1071-1078. doi: 10.1038/nature08467

Jheng, J.-R. R., Ho, J.-Y. Y., and Horng, J.-T. T. (2014). Er Stress, Autophagy, and RNA Viruses. Front. Microbiol. 5, 388. doi: 10.3389/fmicb.2014.00388

Kalra, R. S., Tomar, D., Meena, A. S., and Kandimalla, R. (2020). Sars-Cov-2, ACE2, and Hydroxychloroquine: Cardiovascular Complications, Therapeutics, and Clinical Readouts in the Current Settings. Pathogens 9, 546. doi: 10.3390/ pathogens 9070546

Klionsky, D. J., Eskelinen, E.-L., and Deretic, V. (2014). Autophagosomes, Phagosomes, Autolysosomes, Phagolysosomes, Autophagolysosomes... Wait, I'm Confused. Autophagy 10, 549-551. doi: 10.4161/auto.28448

Knoops, K., Kikkert, M., Worm, S. H. E. V. D., Zevenhoven-Dobbe, J. C., Meer, Y.V. d., Koster, A. J., et al. (2008). SARS-Coronavirus Replication is Supported by a Reticulovesicular Network of Modified Endoplasmic Reticulum. PLoS Biol. 6, e226. doi: 10.1371/journal.pbio.0060226

Krähling, V., Stein, D. A., Spiegel, M., Weber, F., and Mühlberger, E. (2009). Severe Acute Respiratory Syndrome Coronavirus Triggers Apoptosis Via Protein Kinase R But is Resistant to Its Antiviral Activity 7 . J. Virol. 83, 2298-2309. doi: $10.1128 /$ jvi.01245-08

Lee, Y.-R., Kuo, S.-H., Lin, C.-Y., Fu, P.-J., Lin, Y.-S., Yeh, T.-M., et al. (2018). Dengue Virus-Induced Er Stress is Required for Autophagy Activation, Viral Replication, and Pathogenesis Both in Vitro and In Vivo. Sci. Rep-uk 8, 489. doi: 10.1038/s41598-017-18909-3

Liao, Y., Fung, T. S., Huang, M., Fang, S. G., Zhong, Y., and Liu, D. X. (2013). Upregulation of CHOP/GADD153 During Coronavirus Infectious Bronchitis Virus Infection Modulates Apoptosis by Restricting Activation of the Extracellular SignalRegulated Kinase Pathway. J. Virol. 87, 8124-8134. doi: 10.1128/jvi.00626-13 
Lindner, P., Christensen, S. B. B., Nissen, P., Møller, J. V., and Engedal, N. (2020). Cell Death Induced by the ER Stressor Thapsigargin Involves Death Receptor 5, a Non-Autophagic Function of MAP1LC3B, and Distinct Contributions From Unfolded Protein Response Components. Cell communication signaling : CCS 18, 12. doi: 10.1186/s12964-019-0499-Z

Liu, C., Yan, D.-Y. Y., Wang, C., Ma, Z., Deng, Y., Liu, W., et al. (2020). Ire1 Signaling Pathway Mediates Protective Autophagic Response Against Manganese-Induced Neuronal Apoptosis in Vivo and In Vitro. Sci. Total Environ. 712, 136480. doi: 10.1016/j.scitotenv.2019.136480

Maisonnasse, P., Guedj, J., Contreras, V., Behillil, S., Solas, C., Marlin, R., et al. (2020). Hydroxychloroquine Use Against SARS-Cov-2 Infection in NonHuman Primates. Nature 585, 584-587. doi: 10.1038/s41586-020-2558-4

Mauthe, M., Orhon, I., Rocchi, C., Zhou, X., Luhr, M., Hijlkema, K.-J., et al. (2018). Chloroquine Inhibits Autophagic Flux by Decreasing Autophagosome-Lysosome Fusion. Autophagy 14, 1435-1455. doi: 10.1080/15548627.2018.1474314

McQuiston, A., and Diehl, J. A. (2017). Recent Insights Into PERK-Dependent Signaling From the Stressed Endoplasmic Reticulum. F1000research 6, 1897. doi: 10.12688/f1000research.12138.1

Mehrbod, P., Ande, S. R., Alizadeh, J., Rahimizadeh, S., Shariati, A., Malek, H., et al. (2019). The Roles of Apoptosis, Autophagy and Unfolded Protein Response in Arbovirus, Influenza Virus, and HIV Infections. Virulence 10, 376-413. doi: 10.1080/21505594.2019.1605803

Mercer, T. J., Gubas, A., and Tooze, S. A. A. (2018). Molecular Perspective of Mammalian Autophagosome Biogenesis. J. Biol. Chem. 293, 5386-5395. doi: 10.1074/jbc.r117.810366

Mesel-Lemoine, M., Millet, J., Vidalain, P.-O., Law, H., Vabret, A., Lorin, V., et al. (2012). Human Coronavirus Responsible for the Common Cold Massively Kills Dendritic Cells But Not Monocytes. J. Virol. 86, 7577-7587. doi: 10.1128/jvi.00269-12

Minakshi, R., Padhan, K., Rani, M., Khan, N., Ahmad, F., and Jameel, S. (2009). The SARS Coronavirus 3a Protein Causes Endoplasmic Reticulum Stress and Induces Ligand-Independent Downregulation of the Type 1 Interferon Receptor. PLoS One 4, e8342. doi: 10.1371/journal.pone.0008342

Mizushima, N., Levine, B., Cuervo, A. M., and Klionsky, D. J. (2008). Autophagy Fights Disease Through Cellular Self-Digestion. Nature 451, 1069-1075. doi: $10.1038 /$ nature06639

Mohd, H. A., Al-Tawfiq, J. A., and Memish, Z. A. (2016). Middle East Respiratory Syndrome Coronavirus (Mers-Cov) Origin and Animal Reservoir. Virol. J. 13, 87. doi: 10.1186/s12985-016-0544-0

Palmeira, A., Sousa, E., Köseler, A., Sabirli, R., Gören, T., Türkçüer, İ., et al. (2020). Preliminary Virtual Screening Studies to Identify Grp78 Inhibitors Which May Interfere With Sars-Cov-2 Infection. Pharm 13, 132. doi: 10.3390/ph13060132

Prentice, E., Jerome, G. W., Yoshimori, T., Mizushima, N., and Denison, M. R. (2003). Coronavirus Replication Complex Formation Utilizes Components of Cellular Autophagy. J. Biol. Chem. 279, 10136-10141. doi: 10.1074/jbc.m306124200

Rashid, H.-O., Yadav, R. K., Kim, H.-R., and Chae, H.-J. (2015). Er Stress: Autophagy Induction, Inhibition and Selection. Autophagy 11, 1956-1977. doi: 10.1080/15548627.2015.1091141

Reggiori, F., Haan, C. A. M. de, and Molinari, M. (2011). Unconventional Use of LC3 by Coronaviruses Through the Alleged Subversion of the ERAD Tuning Pathway. Viruses 3, 1610-1623. doi: 10.3390/v3091610

Reggiori, F., and Klionsky, D. J. (2005). Autophagosomes: Biogenesis From Scratch? Curr. Opin. Cell Biol. 17, 415-422. doi: 10.1016/j.ceb.2005.06.007

Reggiori, F., Monastyrska, I., Verheije, M. H., Calì, T., Ulasli, M., Bianchi, S., et al. (2010). Coronaviruses Hijack the LC3-I-Positive Edemosomes, ER-Derived Vesicles Exporting Short-Lived ERAD Regulators, for Replication. Cell Host Microbe 7, 500-508. doi: 10.1016/j.chom.2010.05.013

Ríos-Ocampo, W. A., Navas, M.-C., Faber, K. N., Daemen, T., and Moshage, H. (2018). The Cellular Stress Response in Hepatitis C Virus Infection: A Balancing Act to Promote Viral Persistence and Host Cell Survival. Virus Res. 263, 1-8. doi: 10.1016/j.virusres.2018.12.013

Romero-Brey, I., and Bartenschlager, R. (2014). Membranous Replication Factories Induced by Plus-Strand Rna Viruses. Viruses 6, 2826-2857. doi: $10.3390 / \mathrm{v} 6072826$

Rzymski, T., Milani, M., Pike, L., Buffa, F., Mellor, H. R., Winchester, L., et al. (2010). Regulation of Autophagy by ATF4 in Response to Severe Hypoxia. Oncogene 29, 4424-4435. doi: 10.1038/onc.2010.191

Schneider, W. M., Luna, J. M., Hoffmann, H.-H., Sánchez-Rivera, F. J., Leal, A. A., Ashbrook, A. W., et al. (2021). Genome-Scale Identification of SARS-Cov-2 and Pan-Coronavirus Host Factor Networks. Cell 184, 120-132.e14. doi: 10.1016/j.cell.2020.12.006

Senft, D., and Ronai, Z. A. (2015). Upr, Autophagy, and Mitochondria Crosstalk Underlies the ER Stress Response. Trends Biochem. Sci. 40, 141-148. doi: 10.1016/j.tibs.2015.01.002

Senger, M. R., Evangelista, T. C. S., Dantas, R. F., Santana, M. V. da S., Gonçalves, L. C. S., Neto, L. R. de S., et al. (2020). Covid-19: Molecular Targets, Drug Repurposing and New Avenues for Drug Discovery. Memórias Instituto Oswaldo Cruz 115, e200254. doi: 10.1590/0074-02760200254

Shaban, M. S., Müller, C., Mayr-Buro, C., Weiser, H., Albert, B. V., Weber, A., et al. (2020). Inhibiting Coronavirus Replication in Cultured Cells by Chemical Er Stress. Biorxiv 2020, 0826.266304. doi: 10.1101/2020.08.26.266304

Shi, C.-S., Nabar, N. R., Huang, N.-N., and Kehrl, J. H. (2019). Sars-Coronavirus Open Reading Frame-8b Triggers Intracellular Stress Pathways and Activates Nlrp3 Inflammasomes. Cell Death Discov. 5, 101. doi: 10.1038/s41420-0190181-7

Shoulders, M. D., Ryno, L. M., Genereux, J. C., Moresco, J. J., Tu, P. G., Wu, C., et al. (2013). Stress-Independent Activation of XBP1s and/or ATF6 Reveals Three Functionally Diverse Er Proteostasis Environments. Cell Rep. 3, 12791292. doi: 10.1016/j.celrep.2013.03.024

Siqueira, M., Ribeiro, R., and Travassos, L. H. (2018). Autophagy and Its Interaction With Intracellular Bacterial Pathogens. Front. Immunol. 9, 935. doi: 10.3389/fimmu.2018.00935

Smith, M., and Wilkinson, S. (2017). Er Homeostasis and Autophagy. Essays Biochem. 61, 625-635. doi: 10.1042/EBC20170092

Sung, S.-C., Chao, C.-Y., Jeng, K.-S., Yang, J.-Y., and Lai, M. M. C. (2009). The $8 \mathrm{ab}$ Protein of SARS-Cov is a Luminal Er Membrane-Associated Protein and Induces the Activation of ATF6. Virology 387, 402-413. doi: 10.1016/ j.virol.2009.02.021

Tan, X., Thapa, N., Liao, Y., Choi, S., and Anderson, R. A. (2016). Ptdins(4,5)P2 Signaling Regulates ATG14 and Autophagy. Proc. Natl. Acad. Sci. U. S. A. 113, 10896-10901. doi: 10.1073/pnas.1523145113

Touret, F., Gilles, M., Barral, K., Nougairède, A., Helden, J.v., Decroly, E., et al. (2020). In Vitro Screening of a FDA Approved Chemical Library Reveals Potential Inhibitors of SARS-Cov-2 Replication. Sci. Rep-uk 10, 13093. doi: 10.1038/s41598-020-70143-6

Ulferts, R., Imbert, I., Canard, B., and Ziebuhr, J. (2009). Expression and Functions of SARS Coronavirus Replicative Proteins. Molecular Biology of the SARSCoronavirus 75-98. doi: 10.1007/978-3-642-03683-5_6

Urra, H., Henriquez, D. R., Cánovas, J., Villarroel-Campos, D., Carreras-Sureda, A., Pulgar, E., et al. (2018). Irel $\alpha$ Governs Cytoskeleton Remodelling and Cell Migration Through a Direct Interaction With Filamin a. Nat. Cell Biol. 20, 942-953. doi: 10.1038/s41556-018-0141-0

V'kovski, P., Kratzel, A., Steiner, S., Stalder, H., and Thiel, V. (2020). Coronavirus Biology and Replication: Implications for SARS-Cov-2. Nat. Rev. Microbiol. 19, 1-16. doi: 10.1038/s41579-020-00468-6

Vliet, A. R. v., Giordano, F., Gerlo, S., Segura, I., Eygen, S. V., Molenberghs, G., et al. (2017). the Er Stress Sensor Perk Coordinates Er-Plasma Membrane Contact Site Formation Through Interaction With Filamin-a and F-Actin Remodeling. Mol. Cell 65, 885-899.e6. doi: 10.1016/j.molcel.2017.01.020

Wang, X., Liao, Y., Yap, P. L., Png, K. J., Tam, J. P., and Liu, D. X. (2009). Inhibition of Protein Kinase R Activation and Upregulation of GADD34 Expression Play a Synergistic Role in Facilitating Coronavirus Replication by Maintaining De Novo Protein Synthesis in Virus-Infected Cells $\nabla$. J. Virol. 83, 12462-12472. doi: 10.1128/jvi.01546-09

Wang, K., Lu, W., Chen, J., Xie, S., Shi, H., Hsu, H., et al. (2012). Pedv ORF3 Encodes an Ion Channel Protein and Regulates Virus Production. FEBS Lett. 586, 384-391. doi: 10.1016/j.febslet.2012.01.005

Wolff, G., Melia, C. E., Snijder, E. J., and Bárcena, M. (2020). Double-Membrane Vesicles as Platforms for Viral Replication. Trends Microbiol. 28, 1022-1033. doi: 10.1016/j.tim.2020.05.009

Xiao, Y., and Cai, W. (2020). Autophagy and Viral Infection. Adv. Exp. Med. Biol. 1207, 425-432. doi: 10.1007/978-981-15-4272-5_30

Yang, N., and Shen, H.-M. (2020). Targeting the Endocytic Pathway and Autophagy Process as a Novel Therapeutic Strategy in COVID-19. Int. J. Biol. Sci. 16, 1724-1731. doi: 10.7150/ijbs.45498

Yao, R.-Q. Q., Ren, C., Xia, Z.-F. F., and Yao, Y.-M. M. (2021). Organelle-Specific Autophagy in Inflammatory Diseases: A Potential Therapeutic Target 
Underlying the Quality Control of Multiple Organelles. Autophagy 17 (2), 385-401. doi: 10.1080/15548627.2020.1725377

Ye, Z.-W., Yuan, S., Yuen, K.-S., Fung, S.-Y., Chan, C.-P., and Jin, D.-Y. (2020). Zoonotic Origins of Human Coronaviruses. Int. J. Biol. Sci. 16, 1686-1697. doi: $10.7150 /$ ijbs. 45472

Zhao, Z., Thackray, L. B., Miller, B. C., Lynn, T. M., Becker, M. M., Ward, E., et al. (2007). Coronavirus Replication Does Not Require the Autophagy Gene Atg5. Autophagy 3, 581-585. doi: 10.4161/auto.4782

Zou, D., Xu, J., Duan, X., Xu, X., Li, P., Cheng, L., et al. (2019). Porcine Epidemic Diarrhea Virus Orf3 Protein Causes Endoplasmic Reticulum Stress to Facilitate Autophagy. Vet. Microbiol. 235, 209-219. doi: 10.1016/j.vetmic.2019.07.005
Conflict of Interest: The authors declare that the research was conducted in the absence of any commercial or financial relationships that could be construed as a potential conflict of interest.

Copyright (๑) 2021 Prestes, Bruno, Travassos and Carneiro. This is an open-access article distributed under the terms of the Creative Commons Attribution License (CC BY). The use, distribution or reproduction in other forums is permitted, provided the original author(s) and the copyright owner(s) are credited and that the original publication in this journal is cited, in accordance with accepted academic practice. No use, distribution or reproduction is permitted which does not comply with these terms. 